\title{
Comparison of three most frequently used alpha blocker agents in medical expulsive therapy for distal ureteral calculi, result of a retrospective observational study
}

\author{
Aykut Buğra Sentürk ${ }^{1}$, Cemil Aydin ${ }^{1}$, Musa Ekici ${ }^{1}$, Muhammet Yaytokgil ${ }^{2}$, Ali Akkoc ${ }^{3}$, \\ Mehmet Murat Baykam ${ }^{1}$ \\ ${ }^{1}$ Hitit University Corum Training and Research Hospital, Turkey; \\ ${ }^{2}$ Rize State Hospital, Turkey; \\ ${ }^{3}$ Alanya Alattin Keykubat University, Turkey.
}

\begin{abstract}
Summary Purpose: In this study, we compared the effects of three agents frequently used in daily life for medical expulsive therapy.

Materials and methods: A total of 143 patients meeting the criteria were included in the study. Patients were divided into three homogeneous drug groups which were tamsulosin group (n:48), alfuzosin group ( $n: 47)$ and silodosin group (n:48).

The time of stone expulsion, analgesic needs, side effects of the medicine and endoscopic intervention needs of the patients were recorded.

Results: The rate of stone expulsion was $70.8 \%$ (n:34) in tamsulosin group, $70.2 \%$ (n:33) in alfuzosin group, and $75 \%$ (n:36) in silodosin group. No significant difference was observed among the rates of stone expulsion in three groups, and the rates of stone expulsion were similar $(p=0.778)$. The duration of stone expulsion was significantly different in the groups $(p=0.012)$ : the time of stone expulsion for tamsulosin was $2.33 \pm 0.78$ days longer than for Silodosin, indicating a significant difference. There was no significant difference between tamsulosin-alfuzosin and silodosin-alfuzosin (respectively $p=0.147$,

$p=0.925$ ).

Conclusions: The results of this study showed that medical expulsive therapy by using alpha blocker agents is safe and efficacious. This option must be kept in mind for patients who do not ask for surgery as the first-step treatment for eligible patients.
\end{abstract}

KEY WORDS: Urology; Ureter; Stone.

Submitted 13 February 2018; 24 February 2018

\section{INTRODUCTION}

Urolithiasis is one of the most common disorders of urinary tract affecting about $5 \%-10 \%$ of the population. Renal stones are most prevalent between the ages of 20 and 40 years and are three times greater in men than women (1). Women typically excrete more citrate and less calcium than men, which may explain the higher incidence of stone diseases in men. Twenty-two percent of all urinary tract stones are found in ureter, of which $68 \%$ are seen in the distal ureter (2).

The treatment of urinary stones basically varies depending on the anatomic location of the stone, the size of the stone and the factors related with the patient. The treatments of the ureteral calculi are observation, medical expulsive therapy, extracorporeal shock wave and lithotripsy (ESWL), retrograde ureterorenoscopy, antegrade percutaneous ureterorenoscopy, and laparoscopic and open ureterolithotomy (3).

The location and the size of the stone, the availability of the technology, the treatment cost, the experience of the surgeon, and the preference of the patients are considered when a treatment is chosen among the other alternatives (4).

The probability of spontaneous expulsion of the ureteral calculi has two factors: the size of the calculi and the anatomic location of the calculi. According to a metaanalysis, the rate of spontaneous expulsion of the stones smaller than $5 \mathrm{~mm}$ is $68 \%$ while it is $47 \%$ for the stones bigger than $5 \mathrm{~mm}$ and smaller than $10 \mathrm{~mm}$ (5). When anatomic location is considered, it is seen that $71 \%$ of the distal ureteral calculi and $22 \%$ of the proximal ureteral calculi expulse spontaneously (6). Therefore, spontaneous expulsion of the stone protects the patient from surgical intervention, anesthesia risk and additional costs, who does not have infection history and who has pain control and small size of calculi. By this way, with the understanding of the ureter physiology in detail, the concept of medical expulsive therapy has been developed in order to make the spontaneous expulsion of the stone easier.

The purpose of the medical expulsive therapy is to increase the spontaneous probability of the stone expulsion by enabling relaxation in the ureter smooth muscle structure and eventually it reduces the pain level and frequency felt by the patient, shorten the time of stone expulsion, reduces the need of operation, prevents the risk and complications related with the operation and reduces the cost of the treatment. Some main points need attention during the medical expulsive treatment. The most important two factors of them are the location of the calculi in the ureter and the size of the calculi. The maximum upper limit recommended for the treatment of the medical expulsive is $10 \mathrm{~mm}(7)$.

Many treatment alternatives are available for medical expulsive treatment. Calcium channel blockers, alpha blockers, phosphodiesterase type 5 inhibitors and corticosteroids are the most frequently used drugs. In the guide of European Society of Urology, it is mentioned that 
alpha blockers are more successful for medical expulsive treatment and calcium channel blockers are successful only when nifedipine is used for medical expulsive treatment. Also corticosteroids are recommended to be used not alone but with other drugs for medical expulsive treatment purpose (8).

In the various studies, it was shown that phosphodiesterase type 5 inhibitors increase the spontaneous stone expulsion by causing the relaxation of ureter smooth muscles; however, there is no sufficient data for its clinical use (9).

Although alpha adrenergic receptors are available in all ureter segments, these receptors are usually located at distal ureter (10). Density order in distal ureter is alphald $>$ alpha-la>alpha-1b (11).

In this retrospective study, we investigated the effects of three agents frequently used in daily life for medical expulsive therapy on each other.

\section{Materials AND METHOdS}

The study was undertaken retrospectively in accordance with the principles of the Declaration of Helsinki. Between January 2013 and October 2017, the data of 365 patients who were admitted to a polyclinic with distal ureter calculi size between $4-10 \mathrm{~mm}$ were investigated retrospectively.

They were grouped homogeneously in terms of calculi size, patient age and gender. Those patients who had bilateral ureter calculi, severe urinary tract infection, severe colic attack, fever, severe hydronephrosis, renal impairment, history of endoscopic surgery due to ureter calculi, and history of drug which interact with alpha blockers were excluded from the study. Urine analysis, blood urea and creatinine values and complete blood count of all the patients were recorded before the treatment. Those patients who had calculi with the size of 4$10 \mathrm{~mm}$ which were located under the common iliac arteries and confirmed by computarized tomography, and those responding to the analgesic treatment were included in the study. A total of 143 patients meeting the criteria were included in the study. Patients were divided into three homogeneous drug groups which were tamsulosin group (n:48), alfuzosin group (n:47) and silodosin group (n:48)

The patients in tamsulosin group received one dose of 0.4 $\mathrm{mg} /$ day tamsulosin orally, the patients in alfuzosin group received one dose of $10 \mathrm{mg} /$ day alfuzosin orally, and those in silodosin group received one dose of $8 \mathrm{mg} /$ day silodosin orally. In each group, the medical treatment was maintained until the patients expulsed the stone or for four weeks. When the patients had pain, they were administered analgesic.

The time of stone expulsion, analgesic needs, side effects of the drug and endoscopic intervention needs of the patients were recorded. The expulsion of the non-transparent stones was confirmed by ultrasonography and transparent stones were confirmed by unenhanced tomography.

All statistical analysis were performed with SPSS statistical software (Version
22.0, SPSS Inc., Chicago, IL, USA). Descriptive statistics were presented as mean \pm standard deviation (SD) values. Shapiro-Wilk test was used to check for normality of distribution. Patient characteristics in the three age groups were compared using Pearson's chi-squared test in case of discrete variables.

The significance of the difference between three groups were assessed by using one-way analysis of variance (ANOVA) in case of normal data distribution, or KruskalWallis test (non-parametric analysis of variance) in case of non-normal distribution for continuous variables. Bonferroni post hoc test was applied to determine the differences between the pairwise groups. P values $<0.05$ were considered to be statistically significant.

\section{RESULTS}

In each group, the sizes of the stones were similar ( $\mathrm{p}=$ 0.224) (Table 1).

The sizes of the stones were $7.10 \pm 1.80 \mathrm{~mm}$ in tamsulosin group, $6.55 \pm 1.58 \mathrm{~mm}$ in alfuzosin group, and $6.65 \pm 1.57 \mathrm{~mm}$ in silodosin group.

The rate of stone expulsion was $70.8 \%$ (n:34) in tamsulosin group, $70.2 \%$ (n:33) in alfuzosin group, and 75\% (n:36) in silodosin group. No significant difference was observed among the rates of stone expulsion in the three groups, and the rates of stone expulsion were similar $(p=0.778)$. Despite the medical expulsive treatment lasting for four weeks, the rates of ureterorenoscopy operations due to non-expulsing stone was 29.2\% (n:14) in tamsulosin group, $10.6 \%$ (n:14) in alfuzosin group and 25\% (n:12) in silodosin group.

The duration of stone expulsion was $10.41 \pm 3.61$ days in tamsulosin group, $8.87 \pm 3.54$ days in alfuzosin group, and $8.09 \pm 3.66$ days in silodosin group.

The duration of stone expulsion was significantly different in the groups $(p=0.012)$. According to post hoc test results, the difference between silodosin and tamsulosin groups was $(\mathrm{p}=0.010)$. So, the time of stone expulsion in tamsulosin was $2.33 \pm 0.78$ days longer than the one in silodosin, indicating a significant difference.

There was no significant difference between tamsulosinalfuzosin and silodosin-alfuzosin (respectively $\mathrm{p}=0.147$, $\mathrm{p}=0.925$ )

No statistical difference was found between 3 drug groups in terms of frequency of colic attack and analgesic usage (respectively $\mathrm{p}=0.25, \mathrm{p}=0.45$ ). Hypotension which is a major adverse effect of the drug was $8.5 \%$ in tamsulosin group, $4.5 \%$ in silodosin group, and $6.4 \%$ in alfuzosin group. Although retrograde ejaculation was seen more frequently in silodosin group than the other groups, there was no statistical difference $(p=0.35)$.
Table 1.

Demographic values of groups.

\begin{tabular}{|lcccc|}
\hline & Tamsulosin & Alfuzosin & Silodosin & P value \\
\hline Mean age $\pm \mathrm{SD}$ (year) & $40.37 \pm 12.43$ & $41.15 \pm 12.15$ & $41.46 \pm 15.04$ & $p=0.919$ \\
\hline Mean stone diameter $\pm \mathrm{SD}(\mathrm{mm})$ & $7.10 \pm 1.80$ & $6.55 \pm 1.58$ & $6.65 \pm 1.57$ & $p=0.224$ \\
\hline Male/female $(\mathrm{n})$ & $24 / 24$ & $27 / 20$ & $26 / 22$ & $p=0.778$ \\
\hline
\end{tabular}


Figure 1.

Stone expulsion duration in the groups.

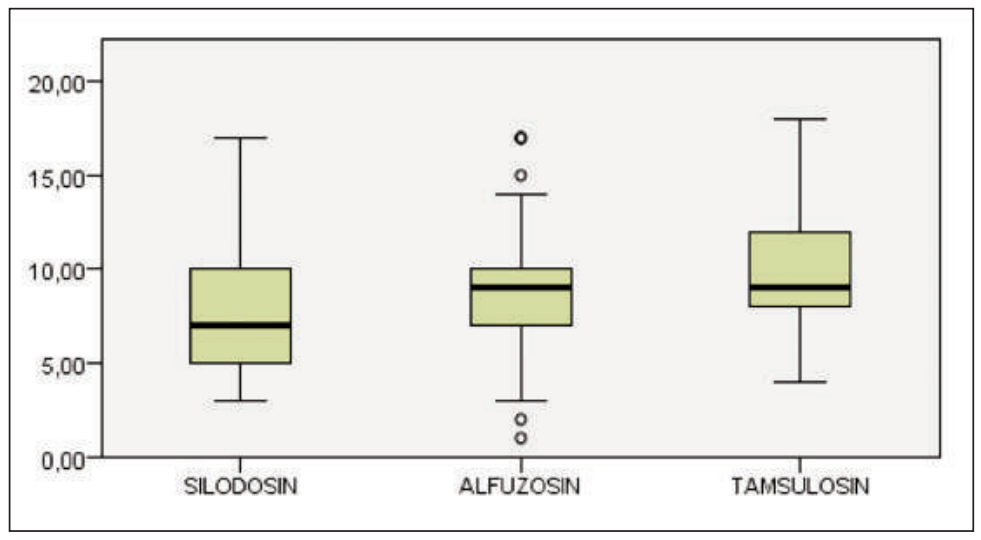

A study comparing the efficacy of silodosin to tamsulosin including 136 patients with proximal ureter stone which are in diameter of 4-10 $\mathrm{mm}$ showed that the patients treated with silodosin demonstrated a significant increase in expulsion rate and a decrease in expulsion duration of lower ureteral stones (61.2 versus 80.3\%) (19). A meta-analysis involving eight publications from Huang $W$ et al. indicated that silodosin was superior to placebo or tamsulosin in the efficacy for distal ureteral calculi treatment with better control of pain (18). Also, a multi-institutional, randomized, double-blinded, placebo-controlled trial from Sur $R L$ et al. reported that silodosin was found to be well tolerated and beneficial in facilitating the expulsion of distal ureteral stones (17).

In our study, we found a similar effect between

\section{Discussion}

Due to risk of complications for ureteral stones less than $10 \mathrm{~mm}$ in size during minimal invasive treatments and their high costs, nowadays the treatment of ureteral stones vary in the direction of conservative treatment. Management of ureteral stones depends on the size, location, number, structure of the stone and presence of the symptoms. Ureteral spasm, ureteral anatomy and mucosal edema by inflammation affect the rate of stone expulsion. Watchful waiting for distal ureteral stones is a good option in patients with no infection, tolerable colic attacks and small stone size. The aim of medical expulsive therapy is to facilitate spontaneous stone expulsion by relaxing ureteral smooth muscle without any disruption of ureteral peristalsis and to reduce the severity of pain of the patient.

This idea depends on good spontaneous expulsion rates of small ureteral stones. Natural spontaneous expulsion rate of distal ureteric calculi is $68 \%$ for stones less than 5 $\mathrm{mm}$ in size. And this rate is about $47 \%$ for stones with sizes between 5 and $10 \mathrm{~mm}$ (5). Besides of the size, the localization of the calculi is also an important factor for spontaneous expulsion. The spontaneous expulsion rate of proximal ureter stone is $21 \%$, of middle ureter stone $46 \%$, and of distal ureteral stone $71 \%$ (6).

Alpha-1 receptors have been classified into three subtypes, which are alpha-1A, alpha-1B and alpha-1D. Alpha-1D and alpha-1A are the most common adrenoceptors found in the ureter (12) and the distributions of these receptors are alpha-1D > alpha-1A > alpha-1B (11). Alpha-1D receptors are found predominantly in the intramural ureter and detrusor muscle and they are the target of medical expulsive therapy as they are found generally in the distal ureter (13). Itoh et al. reported that the distal part of the ureter expresses the higher amount of alpha-1 adrenoceptor than the other parts. Also it was demonstrated that alpha-1D adrenoceptor mRNA is much more common than alpha-1A adrenoceptor mRNA in each part of the ureter. Therefore, alpha-1D adrenoceptor blocker can be more useful than alpha 1A adrenoceptor blocker to facilitate expulsion of ureteral calculi according to their study (11). But in contrast, Tatemichi et al. reported that ureteral motility is medicated more commonly by alpha $1 \mathrm{~A}$ adrenoceptors (14). silodosin and tamsulosin groups in terms of stone expulsion $(\mathrm{p}=0.010)$. Stone expulsion duration was $10.41+$ 3.61 days in tamsulosin group and $8.09+3.66$ days in silodosin group. The stone expulsion duration of tamsulosin was significantly longer than the duration with silodosin (2.33 \pm 0.78 days). As mentioned in similar studies, we consider that this finding is associated with the selective alpha 1-A adenoceptor antagonist effect of silodosin rather than the alpha-1 adrenoceptor antagonist effect of tamsulosin. We also did not found any statistical difference between tamsulosin-alfuzosin and silodosin-alfuzosin in terms stone expulsion duration $(\mathrm{p}=0.147, \mathrm{p}=0.925$ respectively).

In the study of Imperatore $V$ et al., it was reported that both tamsulosin and silodosin are equally effective as medical expulsive treatment (MET) for distal ureteral calculi sized $<10 \mathrm{~mm}$. Stone-expulsion rate was $88 \%$ in silodosin group and $82 \%$ in tamsulosin group (20). Similarly, while stone expulsion rate was $70.8 \%$ in tamsulosin group, $70.2 \%$ in alfuzosin group and $75 \%$ in silodosin group in our study, we found no statistical difference between three groups in terms of stone expulsion rates $(p=0.778)$.

Increase in intraureteral pressure due to obstruction causes colic pain attacks. Alpha blockers which are used predominantly for stone expulsion may also decrease analgesic drug usage by expulsion of ureteral calculi (16). Kumar et al. reported that stone expulsion by an alpha 1 adrenoceptor on the obstructed ureter is facilitated by increasing the intaureteral pressure gradient around the stone and decreasing peristalsis below the ureter (13) and alpha blockade may decrease ureteric colic attacks by blocking C fibers which are responsible for pain (15).

Although we could not find any statistical difference between three groups in terms of the frequency of colic attack in our study $(p=0.45)$. The medical expulsive treatment should be discontinued in case of severe urinary infection and hydronephrosis, and endoscopic surgery should be considered. In our study, the rate of patients who needed endoscopic procedure due to nonexpulsing stones was $29.2 \%$ (n:14) in tamsulosin group, $10.6 \%$ (n:14) in alfuzosin group, and 25\% (n:12) in silodosin group. Medical expulsive therapy is a cost-effective non-surgical treatment for ureteral calculi less than 10 
$\mathrm{mm}$ in size. Several studies showed that alpha-1 adrenoceptor blockers can facilitate spontaneous passage of distal ureteral calculi with minimal side effects. A study from Bensalah $\mathrm{K}$ et al. reported that medical expulsive therapy using tamsulosin resulted in a cost advantage for 1,132 USD over observation in USA. Since the cost of tamsulosin is only 2.08 USD per day whereas the estimated cost of ureteroscopy is 4973 USD in USA (4).

According to a systematic review and meta-analysis based on 21 studies, of which the main topic was to understand the effect of medical expulsive treatment (MET) of ureter stone, by Picozzi SC et al., it was reported that medical expulsive therapy should be offered to patients who are complaining about distal ureteral calculi (21).

Our study have some limitations. One of them is that we do not have a control group since the main objective of the study was to compare the effects of these three different type of alpha blockers. Other limitation was the small number of the samples. However, the results of the power analysis during the design of the study showed that current numbers were not statistically problematic.

\section{Conclusion}

No significant difference was found between the three groups in terms of the rate of stone expulsion ( $p=$ 0.778 ). However, the duration of stone expulsion had a significant difference among the groups $(\mathrm{p}=0.012)$. Stone expulsion duration for tamsulosin was $2.33 \pm 0.78$ days longer than for silodosin, which is a considerable difference $(p=0.010)$. There was no significant difference between tamsulusin-alfuzosin and silodosin-alfuzosin $(\mathrm{p}=0.147$ and $\mathrm{p}=0.925$, respectively). The results of this study showed that medical expulsive therapy by using alpha blocker agents are safe and efficacious.

This option must be kept in mind who do not ask for surgery as the first-step treatment for eligible patients.

\section{REFERENCES}

1. Manglaviti G, Tresoldi S, Guerrer CS, et al. In vivo evaluation of the chemical composition of urinary stones using dual-energy CT. AJR Am J Roentgenol. 2011; 197:W76-83.

2. Hollingsworth JM, Rogers MA, Kaufman SR, et al. Medical therapy to facilitate urinary stone passage: a meta-analysis. Lancet. 2006; 368:1171-1179.

3. Ergun $O$, Gonen M. Üriner sistem tas hastalıginda medikal ekspulsif tedavi: Kime, nasil, ne kadar? Endoüroloji bülteni 2014; 7:74-76.

4. Bensalah K, Pearle M, Lotan Y. Cost-effectiveness of medical expulsive therapy using alpha-blockers for the treatment of distal ureteral stones. Eur Urol. 2008; 53:411-8.

5. Preminger GM, Tiselius HG, Assimos DG, et al. EAU/AUA Nephrolithiasis Guideline Panel. 2007 guideline for the management of ureteral calculi. J Urol. 2007; 178:2418-34.

6. Morse RM, Resnick MI. Ureteral calculi: natural history and treatment in an era of advanced technology. J Urol. 1991; 145:263-5.

7. Singh A, Alter HJ, Littlepage A. A systematic review of medical therapy to facilitate passage of ureteral calculi. Ann Emerg Med. 2007; 50:552-63

8. Türk C, Petrík A, Sarica K, et al. EAU Guidelines on Diagnosis and Conservative Management of Urolithiasis. Eur Urol. 2016; 69:468-74

9. Gratzke C, Uckert S, Kedia G, et al. In vitro effects of PDE5 inhibitors sildenafil, vardenafil and tadalafil on isolated human ureteral smooth muscle: a basic research approach. Urol Res. 2007; $35: 49-54$

10. Atan A. Medikal Ekspulsif Tedavi: Yeni Olan Nedir? Endoüroloji Bülteni. 2015; 8:78-80.

11. Itoh Y, Kojima Y, Yasui T, Tet al. Examination of alpha 1 adrenoceptor subtypes in the human ureter. Int J Urol. 2007; 14:749-53.

12.Sigala S, Dellabela M, Milanese G, et al. Evidence for the presence of alpha ladrenoceptor subtypes in the human ureter. Neurourol Urodyn. 2005; 24:142-148.

13. Kumar S, Kurdia KC, Ganesamoni R, et al. Randomized controlled trial to compare the safety and efficacy of naftopidil and tamsulosin as medical expulsive therapy in combination with prednisolone for distal ureteral stones. Korean J Urol. 2013; 54:311-5.

14. Tatemichi S, Tomiyama Y, Maruyama I, et al. Uroselectivity in male dogs of silodosin (KMD-3213), a novel drug for the obstructive component of benign prostatic hyperplasia. Neurourol Urodyn. 2006; 25:792-9; discussion 800-1.

15. Kinnman E, Nygards EB, Hansson P. Peripheral alpha-adrenoceptors are involved in the development of capsaicin induced ongoing and stimulus evoked pain in humans. Pain. 1997; 69:79-85.

16. Kumar S, Jayant K, Agrawal MM, et al. Role of tamsulosin tadalafil, and silodosin as the medical expulsive therapy in lower ureteric stone: a randomized trial (a pilot study). Urology. 2015; 85:59-63.

17. Sur RL, Shore N, L'Esperance J, et al. Silodosin to facilitate passage of ureteral stones: a multi-institutional, randomized, doubleblinded, placebo-controlled trial. Eur Urol. 2015; 67:959-64.

18. Huang W, Xue P, Zong H, Zhang Y. Efficacy and safety of silodosin in the medical expulsion therapy for distal ureteral calculi: a systematic review and meta-analysis. Br J Clin Pharmacol. 2016; 81:13-22.

19. Dell'Atti L. Silodosin versus tamsulosin as medical expulsive therapy for distal ureteral stones: a prospective randomized study. Urologia. 2015; 82:54-7.

20. Imperatore V, Fusco F, Creta M, et al. Medical expulsive therapy for distal ureteric stones: tamsulosin versus silodosin. Arch Ital Urol Androl. 2014; 86:103-7.

21. Picozzi SC, Marenghi C, Casellato S, et al. Management of ureteral calculi and medical expulsive therapy in emergency departments. J Emerg Trauma Shock. 2011; 4:70-6.

\section{Correspondence}

Aykut Buğra Sentürk, MD (Corresponding Author) aykutbugra@gmail.com

Cemil Aydin, MD

cemilaydin78@yahoo.com.tr

Musa Ekici, MD

musaekici40@gmail.com

Hitit University Corum Training and Research Hospital, Turkey 